\title{
Introduction of STEM for stress analysis in statically determined bodies
}

\author{
A. N. Galybin \\ Wessex Institute of Technology, Southampton, UK
}

\begin{abstract}
This article presents a novel approach to the identification of stress states in statically determined bodies. The approach is based on the stress trajectory concept and therefore it is referred to as the stress trajectories element method, (STEM). Three different variants of STEM are presented and some problems associated with these are posed.
\end{abstract}

Keywords: boundary value problems, stress trajectories, numerical methods.

\section{Introduction}

This article is an introduction to a novel numerical method that is currently under development in Wessex Institute of Technology. The method is aimed at the identification of stresses in statically determined bodies by employing stress trajectories.

The concept of stress trajectories comes from photoelasticity, therefore one can adopt the following definition due to Frocht [1]: Stress trajectories are curves the tangents to which represent the directions of one of the principal stresses at the points of tangency. A single stress trajectory is also called an isostatic or a line of principal stresses.

Stresses at each point inside a continuous body represent a second-rank tensor which components satisfy differential equations of equilibrium, DEE, and certain constitutive equations. The latter is of theoretical and/or experimental nature, it is often called "rheology", however only statically determined bodies are considered in this paper. These constitute a broad class and some examples are found in engineering:

- in elasticity, the laplacian applied to the first invariant of the stress tensor should vanish (if body forces are neglected); 
- $\quad$ in ideal plasticity, the deviator of the stress tensor is a constant;

- in granular medium, certain linear relationships between the mean stresses and the stress deviator should be fulfilled;

- in rock mechanics non-linear relationships are frequently used.

Classical formulations of boundary value problems, BVPs, for a statically determined body include boundary conditions, BCs, posed in terms of stresses (or other quantities that can be related to stresses one-to-one) on the whole boundary of the body while the number of boundary conditions coincides with the dimension of the body. Under these restrictions, BVPs are usually wellposed, i.e. have unique and stable (with respect to small perturbations in BCs) solutions. The well-posed nature is vital in modern numerical techniques that have been well developed and implemented into computer programs such as finite element methods, boundary element methods, different particle codes and hybrid programs. However, there are a variety of ill-posed problems that are generally defined as problems with non-unique or unstable solutions, [2]. This paper deals with the problems that can be either well- or ill-posed depending on what information about stress trajectories is supplied. Based on theoretical investigations, a universal numerical method is proposed addressing the ill-posed formulations. Stress trajectories of different families are used to form elements or to introduce assumptions within the elements of chosen shapes, therefore the method is further referred to as the stress trajectories element method, STEM.

\section{Lame-Maxwell equations of equilibrium}

The problems considered are classified with respect to information known in regard to the stress trajectories that can be given: $(a)$ everywhere in a domain; (b) at discrete points; or (c) on the boundary of a domain.

In all cases it is assumed that the domain is in equilibrium, therefore the DEE are valid at each point of the domain including its boundary. These can be presented in different forms, for instance, in the Lame-Maxwell form [3]:

$$
\begin{gathered}
\frac{\partial \sigma_{1}}{\partial s_{1}}+\frac{\sigma_{1}-\sigma_{2}}{\rho_{23}}+\frac{\sigma_{1}-\sigma_{3}}{\rho_{32}}+S_{1}=0, \quad \frac{\partial \sigma_{2}}{\partial s_{2}}+\frac{\sigma_{2}-\sigma_{3}}{\rho_{31}}+\frac{\sigma_{2}-\sigma_{1}}{\rho_{13}}+S_{2}=0 \\
\frac{\partial \sigma_{3}}{\partial s_{3}}+\frac{\sigma_{3}-\sigma_{1}}{\rho_{12}}+\frac{\sigma_{3}-\sigma_{2}}{\rho_{21}}+S_{3}=0
\end{gathered}
$$

Here $\sigma_{\mathrm{k}}$ is a principal stress along the $k$-th stress trajectory (isostatic), (all $\sigma_{\mathrm{k}}$ coincide with eigenvalues of the stress tensor), $s_{\mathrm{k}}$ is a coordinate (arc length) along the $k$-th isostatic, $\rho_{\mathrm{ji}}$ is radius of curvature of the $i$-th isostatic in the plane perpendicular to the $j$-th isostatic; $S_{k}$ is a projection of body forces onto the $k$-th isostatic. In order to distinguish different families of the stress trajectories it is assumed that $\sigma_{3} \leq \sigma_{2} \leq \sigma_{1}$.

For plane problems there are two independent DEE. We further focus on this case that demonstrate all features of the proposed techniques. If body forces are absent than the Lame-Maxwell equations assume the following form 


$$
\frac{\partial \sigma_{1}}{\partial s_{1}}+\frac{\sigma_{1}-\sigma_{2}}{\rho_{2}}+=0, \quad \frac{\partial \sigma_{2}}{\partial s_{2}}+\frac{\sigma_{1}-\sigma_{2}}{\rho_{1}}=0
$$

where the curvature is found as $\rho_{\mathrm{k}}^{-1}=\partial \theta_{\mathrm{k}} / \partial s_{\mathrm{k}}\left(\theta_{\mathrm{k}}\right.$ is inclination of $\sigma_{k}$ in a reference coordinate frame).

Problems associated with the essentially different situations $(a)-(c)$ are discussed in the next sections as well as the different variants of STEM associated with different data. These are summarised in Fig. 1 below.
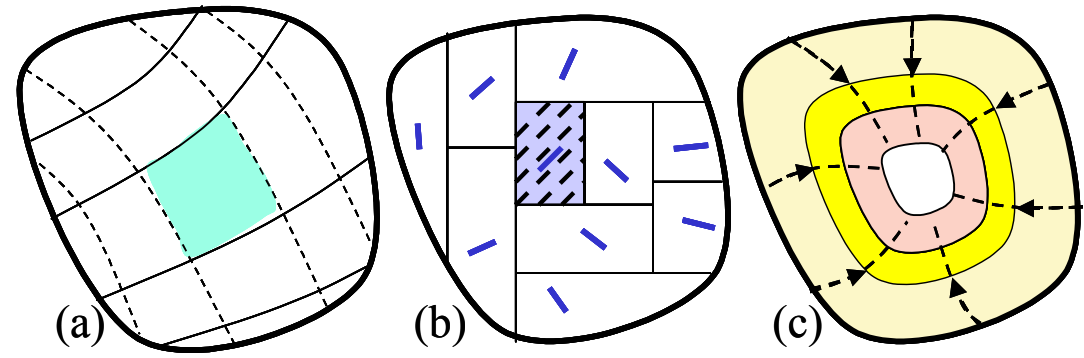

Figure 1: Variants of STEM for different types of data: (a) - trajectories are known, mesh consists of elements formed by trajectories of different families; (b) - data at discrete points, prescribed or adaptive mesh with homogeneous trajectories within elements; (c) - data known on the boundary, adaptive mesh with elements developing from boundary.

\section{Stress trajectories are known everywhere in the domain}

In this case the curvatures of stress trajectories are also known everywhere in the domain considered. Therefore, equations (2) represent a closed system of partial differential equations for the determination of principal stresses. It is important that the knowledge of constitutive equations is unnecessary in order to obtain unique solution of this system. There is a routine operation very well known in photoelasticity, so called, separation of principal stresses, which provides reconstruction of the stress field on the basis of trajectory patterns. However, the knowledge of rheology yields an overspecified formulation.

\subsection{No constitutive equations are given}

It is evident that (2) is of hyperbolic type with characteristics coinciding with stress trajectories, e.g. [4]. Thus, 3 types of classical BVPs can be considered:

- Cauchy BVP assumes BCs given on a line that is not a isostatic;

- Goursat BVP assumes BCs given on isostatics of two different families;

- mixed type of two above.

All three BVPs are well known in partial differential equations. However, one more type has to be mentioned: BCs are posed on the boundary that coincides 
with the stress trajectory of one family (trajectories of the other family are perpendicular to the boundary). Mechanical example for this case is a body which surface is subjected to normal load only (no shear stress). It is clear that different distributions of normal loads on the boundary produce different trajectory patterns within the domain and therefore solutions may exist. For any linear cases (e.g. elasticity) it is also evident that the pattern remains the same if one multiplies the stress magnitudes on the boundary by a non-zero number, which indicates non-uniqueness. Thus, investigation of solvability and uniqueness is one of the tasks of the BVP of this type.

\subsection{Trajectories are known with constitutive equations}

When trajectories are known together with rheology, this results in overspecified system of equations. For instance, the following constitutive equations should be fulfilled for common rheologies

$$
\begin{aligned}
& \text { elasticity: } \Delta\left(\sigma_{1}+\sigma_{2}\right)=0 \\
& \text { ideal plasticity: } \sigma_{1}-\sigma_{2}=2 \tau_{y} \\
& \text { granular media: }\left|\sigma_{1}-\sigma_{2}\right|+\left(\sigma_{1}+\sigma_{2}\right) \tan \phi=\tau_{c}, \quad \sigma_{1}+\sigma_{2} \leq 0
\end{aligned}
$$

Here $\Delta$ is laplacian, $\tau_{\mathrm{y}}$ is yielding limit, $\phi$ is frictional angle and $\tau_{\mathrm{c}}$ is cohesion. For instance, in the case of elasticity equations and (2) and (3) provide uniqueness of the stress field if boundary tractions are specified. Therefore, stress trajectories found from the solution are uniquely determined but they may differ from the given ones. Although in general the problem has no solution, this example poses certain questions regarding consistency of trajectories and rheology, for instance, as follows:

- determine stresses in the body including its boundary provided that rheology and trajectories are consistent;

- given a trajectory pattern, determine whether it can be realised in a body of given rheology with certain restrictions (e.g., boundedness of stresses);

- determine types of trajectory patterns that are consistent with different rheologies.

Consistency in the case of elasticity has been investigated by Mukhamediev and Galybin $[5,6]$ who proved that with the exception of some special cases the complete stress tensor is reconstructed from stress orientations non-uniquely. However, the degree of non-uniqueness is determined. Thus, the solution for the 2D stress deviator can have a multiplier that is either a constant (if no restrictions on stress trajectories are imposed) or a real valued bi-holomorphic function (if the angle of inclination of the stress orientations is a harmonic function of coordinates, in this case 5 real constants are arbitrary).

\subsection{Homogeneous trajectories}

Let us consider the case of homogeneous trajectories that seems to be consistent with all rheologies at least with those mentioned above. In fact, due to $\rho_{\mathrm{k}}=\infty$ the general solution of (2) takes the form $\sigma_{1}=\sigma_{1}\left(s_{2}\right), \sigma_{2}=\sigma_{2}\left(s_{1}\right)$, and hence either of the conditions (3)-(5) can be satisfied by proper choice of arbitrary functions 
$\sigma_{1}\left(s_{2}\right)$ and $\sigma_{2}\left(s_{1}\right)$. However, classes of possible stress fields related to homogeneous trajectories are narrow. In elasticity the only allowable stress fields have the form

$$
\sigma_{1}\left(s_{2}\right)=a s_{2}^{2}+b s_{2}+c, \quad \sigma_{2}\left(s_{1}\right)=-a s_{1}^{2}+d s_{1}+e
$$

where $a, b, c, d, e$ are arbitrary real constants. For the cases of ideal plasticity and granular media the difference $\sigma_{1}-\sigma_{2}$ is a specific constant, hence, one arbitrary constant enters into the general solution that constitutes homogeneity of stresses.

\subsection{First variant of STEM}

When trajectories and rheology are consistent, the problem of stress determination inside the body is the primary task. It is not a BVP, and, as evident from the example for homogeneous trajectories, solution of this problem is nonunique in the general case but depends linearly on a number of arbitrary constants. This circumstance leads to the idea of introduction of a numerical method that is somewhat similar to FEM but with the mesh composed of elements bounded by trajectories of different families, Fig.1a. In this case, two unknown principal stresses (functions $\sigma_{1}$ and $\sigma_{2}$ ) should be determined within each element. Approximation of these functions within elements should be consistent with rheology, which, similarly to the example for homogeneous trajectories, imposes certain forms of approximations that depend on sought parameters (e.g. coefficients of approximation polynomials). Furthermore, it is evident that other restrictions, e.g. continuity of principal stresses across adjacent elements, decrease the number of independent parameters that have to be determined. On one hand, a certain number of parameters will remain undetermined until additional data are attracted, e.g. stresses magnitudes at discrete points. On the other hand, this approach addresses the ill-posed nature of the problem and allows one to determine the number of conditions that has to be additionally imposed in order to find a unique stress field. It has been emphasised that the problem in this formulation is not a BVP, which means that BCs cannot be specified independently on each element of the boundary. In particular, this also means that none of existing numerical methods (neither FEM nor BEM) are capable of solving the problems with given stress trajectories.

\section{Stress orientations are known at discrete points}

\subsection{Global reconstruction of stresses from discrete stress orientations}

A typical approach in this case assumes interpolation using different methods. Examples are found in geophysical applications, e.g. [7-10]. The major defect of this approach is that the pattern of stress trajectories obtained by interpolation is not necessarily consistent with rheology introduced when one attempts to recover the complete stress tensor. Moreover, different interpolation techniques apparently lead to different rheologies; therefore, the choice of interpolation methods may be in conflict with physical meaning. 
Consistent approaches have to take rheology into account at the stage of interpolation by employing the Trefftz method. This means that a global solution for the whole domain is sough as a linear combination of independent functions satisfying the governing equations. The problem appears to be a minimisation problem in which calculated stress orientations provide the best fit to data. Its solution is non-unique: although stress trajectories can be found uniquely, stress magnitudes are determined with some degree of arbitrariness. This approach has been suggested in [11-13] and tested for plane elasticity.

\subsection{Problems to be investigated}

\subsubsection{Singular (isotropic) points in stress trajectory fields}

If orientations of principal stresses (principal directions) are known at a dense net of nodes inside the domain, a smooth field of stress trajectories could be obtained by standard interpolation methods (with possible inconsistence with rheology as explained in the previous subsection). However, the direct conversion can be significantly complicated by the presence of singular points where the stress deviator vanishes and stress orientations are unidentified $\left(\sigma_{1}=\sigma_{2}\right)$, which suggests the lack of smoothness of the trajectory field. It is a serious obstacle for STEM development. The existence of singular points is well known in photoelasticity (where they are referred to as isotropic points), also numerical calculations of stress trajectories shows the presence of these points in different configurations, see recent results of experimental and numerical investigations by Joussineaua et al [14]. Two types of isotropic points are usually distinguished in photoelasticity although the existence of isotropic points of higher order is theoretically possible [15]. These points can be classified in accordance with the asymptotic behaviour of the stress deviator function [16]. Both these types may appear simultaneously.

The identification of singular points is a separate task that requires special attention. As far as elastic domains are concerned the problem of stress tensor identification from discrete principal orientations can be solved simultaneously with the problem of trajectory field identification. Moreover, the singular points of different types can also be found simultaneously, see examples presented in [11], which demonstrate that for elastic medium all types of singular points (interlocking type, non-interlocking type [15] and points where stresses have infinite gradient) can be recovered with sufficient accuracy.

\subsubsection{Other problems}

Apart from singular points the following main problems should be addressed:

- investigation of correspondence between interpolation methods and rheology;

- data analysis for the determination of optimal number and types of basis functions in particular cases;

- stability of solutions for different basis functions;

- arbitrariness in the stress tensor for different rheologies;

- development of a variant of the STEM method. 


\subsection{Second variant of STEM}

A possible approach to the latter problem is demonstrated in Fig 1(b). Given that stress orientations are more or less uniformly distributed within the body, it is proposed to introduce such a mesh in which each element contains at least a datum. After that the assumption of homogeneity of stress trajectories within the element can be accepted, which leads to specific expressions for stresses within the element, e.g. (3) for the case of elasticity. Therefore, the total number of parameters to be determined is equal to the number of elements times the number of parameters specific for each rheology. However, the number of independent parameters is much less (it is found from the analysis of arbitrariness in the stress tensor for different rheologies), which means that additional conditions have to be imposed that connects parameters in adjacent elements (e.g., continuity of stress vector). This procedure leaves several parameters to be identified from additional data and all comments made for the STEM in the case of known trajectories remain.

It is, however, impossible to provide continuity of all stress components. At least one of the stress characteristics may be discontinuous across boundaries of adjacent elements. This is a consequence of the introduction of sharp corners in trajectories. For homogeneous domains, continuity of stresses can be prescribed in average or at the vertices of the elements; what assumption is better has to be investigated. In piecewise homogeneous domains the jump of tangential stresses on the interfaces is not zero, therefore if trajectories kink an interface the principal stresses also suffer jumps across the interface satisfying the following conditions (continuity of the stress vector)

$$
\begin{gathered}
\left(\sigma_{1}^{+}-\sigma_{2}^{+}\right) \sin 2 \theta^{+}=\left(\sigma_{1}^{-}-\sigma_{2}^{-}\right) \sin 2 \theta^{-}, \quad \sigma_{2}^{ \pm} \leq \sigma_{1}^{ \pm} \\
\sigma_{1}^{+}+\sigma_{2}^{+}+\left(\sigma_{1}^{+}-\sigma_{2}^{+}\right) \cos 2 \theta^{+}=\sigma_{1}^{-}+\sigma_{2}^{-}+\left(\sigma_{1}^{-}-\sigma_{2}^{-}\right) \cos 2 \theta^{-}
\end{gathered}
$$

where " \pm " referrer to stress tensor characteristics in two adjacent elements. It follows from the first expression in (7) that the angles $\theta^{+}$and $\theta^{-}$have the same sign $(-\pi / 2<\theta \leq \pi / 2)$. Fig 2 illustrates admissible and non-admissible trajectories in two adjacent elements with a common rectilinear interface.
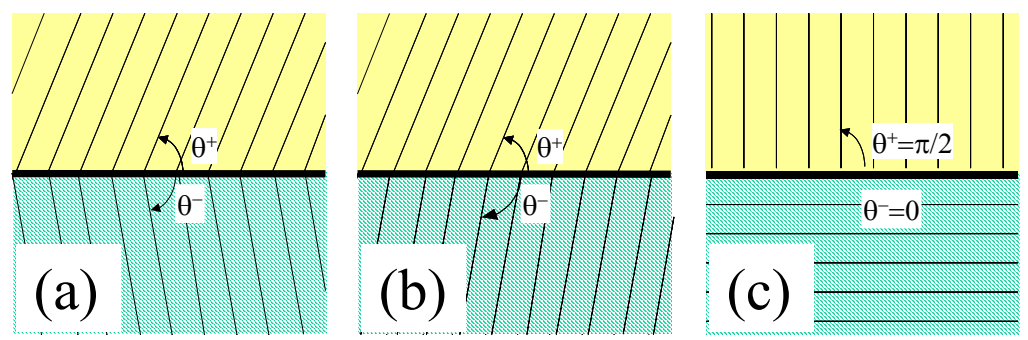

Figure 2: Examples of non-admissible (a) and admissible (b)-(c) stress trajectories of one family. 


\section{Stress orientations given on the boundary}

\subsection{Non-uniqueness of solutions}

It is evident that orientations of principal stresses remain if any constant mean stress is superimposed on a particular solution of DEE or if the stress deviator is multiplied by any positive constant (equilibrium also remains). This indicates non-uniqueness in the BVP formulated in terms of given principal directions (of the stress tensor) on the boundary. However the number of possible solutions is finite if this $\mathrm{BC}$ is complemented by an additional $\mathrm{BC}$, say by continuity of the stress vector. One type of $\mathrm{BC}$ for plane elastic body has been investigated in [17], where the curvatures of stress trajectories have been used as the second condition. It has been shown that this BVP can have finite number of linearly independent solutions (stress states) or have no solutions, which depends on, socalled, index of the problem that is determined from the analysis of the principal directions on the boundary (curvatures of stress trajectories do not affect the index). The number of solutions is uniquely identified by the index, which suggests that the use of other types of the second BC will not make the problem well-posed. This hypothesis has to be thoroughly investigated by analysing different BCs for different rheologies.

\subsection{Third variant of STEM}

Another major task assumes the formulation of a variant of the STEM method for different BVPs. An adaptive mesh with elements developing from the boundary (Fig.1(c)) seems to be the best option because at each step directed inwards the stress orientations are known, therefore trajectories are approximated by tangents, which eventually lead to a piecewise linear system of stress trajectories within the body. As has been mentioned, non-uniqueness and possible instability of solutions considerably restricts the direct application of conventional numerical methods (as FEM or BEM). In the STEM approach the investigation of stability is amongst the major tasks.

\subsection{Applications}

Geophysical applications include determination of stresses in stable blocks of the earth's crust. Here BVPs formulated in terms of stress orientations are of great significance. Stress orientations around the globe are always known near margins of tectonic plates, while data within some plates are limited. For instance, in Antarctic plate stress orientations are mostly known on its boundary, which makes application of conventional back analysis for the identification of tectonic stresses in this plate impossible. However, it has been reported [12] that the approach based on the Trefftz method for complex potentials in elasticity followed by minimisation technique provides stable results in reconstruction of the stress trajectories within Antarctica.

A numerical example for the identification of possible elastic fields within the Antarctic plate is shown in Fig. 3. Data include 170 stress orientation 
measurements shown by segments in Fig. 1(a) (supplied by the WSM project). Continuity of tractions across the boundary have been assumed.
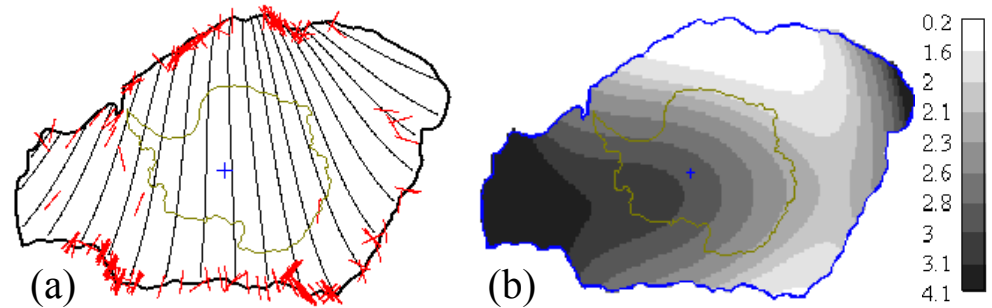

Figure 3: Elastic stresses in the Antarctic plate: stress trajectories (a) and normalised maximum shear stress (b).

There are cases when direct data on stress measurements are unavailable at all, for instance, in crusts of other planets, although investigation of stresses is significant for modelling tectonics, e.g. [18-19]. The only reliable information in these cases is obtained from observations of fracture patterns on the surface. This can be used as an input to identify the orientations of principal stresses, which eventually will lead to mathematical formulations in terms of principal orientations.

At small scales there is a strong influence of measuring devises, e.g. indenters. Therefore the development of non-direct methods for small-scale measurements is also vital as for remote stress field determination mentioned above.

\section{Conclusive remarks and summary}

This article presents main tasks in the programme for the development of the STEM for stress identification in statically determined bodies. Different variants of STEM are proposed addressing three main types of data. The summary of the problems is presented below.

Case 1. Stress trajectories known on boundary with

Unknown Rheology: BVPs for hyperbolic DEE (Cauchy, Goursat, mixed and non-classical)

Given Rheology: overspecified problems, consistency of rheology and trajectories, investigation of solvability.

Case 2. Stress trajectories known at discrete points with given rheology: illposed and minimisation problems (not a BVP), no uniqueness.

Case 3. Stress trajectories known everywhere in domain with given rheology: extra $\mathrm{BC}$ required, ill-posed BVP with non-unique solutions (solvability depends upon the index that can be determined from BCs).

\section{Acknowledgement}

The author acknowledges the support of EPSRC through Research Grant $\mathrm{EP} / \mathrm{E} 032494 / 1$. 


\section{References}

[1] Frocht, M.M. Photoelasticity, Vol. 1. Wiley, New York, 1941

[2] Tikhonov, A.N. and V.Y. Arsenin. Solution of Ill-Posed Problems, New York: Winston, Wiley, 1977.

[3] Papkovich, P.F. Theory of elasticity. Oborongiz, Moscow, 1939.

[4] Mukhamediev, ShA. 1991. Retrieving field of stress tensor in crustal blocks. Izvestiya Earth Physics. 27: 370-7.

[5] Mukhamediev, Sh.A. and A.N. Galybin, 2004. Solution of a plane elastic problem with given trajectories of the principal stresses. Doklady Physics. 49 (5), 311-314

[6] Mukhamediev, Sh.A. and A.N. Galybin, 2007. Determination of Stresses from the Stress Trajectory Pattern in a Plane Elastic Domain. Math. Mech. of Solids, 12, 75-106

[7] Hansen K.M. and V.S. Mount, 1990. Smoothing and extrapolation of crustal stress orientation measurements J.Geophys. Res. 95(B), 1155-1165

[8] Lee, J.-C. and J. Angelier 1994. Paleostress trajectory maps based on the results of local determinations: the "Lissage" program. Computers and Geosciences. 20, 161-191

[9] Bergerat, F. and J. Angelier, 1998. Fault systems and paleostresses in the Vestfirdir Peninsula. Geodinamica Acta (Paris) 11 (2-3), 105-118.

[10] Badawy, A. and F. Horvath, 1999. Recent stress field of the Sinai subplate region. Tectonophysics. 304, 385-403

[11] Galybin, A.N. and Sh.A. Mukhamediev, 2004. Determination of elastic stresses from discrete data on stress orientations. Int. Journal of Solids and Structures. 41 (18-19), 5125-5142

[12] Galybin, A.N. and Sh.A. Mukhamediev, 2004. On the problem of stress reconstruction from discrete orientations of principal stresses. Bollettino di Geofisica Teorica ed Applicata. 45 (1) supplement, 338-342

[13] Mukhamediev, Sh.A., A.N. Galybin and B.H.G. Brady, 2006. Determination of stress fields in elastic lithosphere by methods based on stress orientations. Int Journal of Rock Mechanics and Mining Sciences. 43 (1), 66-88

[14] Joussineaua, G de, J-P Petit and B. D.M. Gauthier, 2003. Photoelastic and numerical investigation of stress distributions around fault models under biaxial compressive loading conditions. Tectonophysics 363, 19- 43

[15] Kuske, A.\& G. Robertson Photoelastic stress analysis. Wiley, London 1974

[16] Karakin A.V. and Sh.A. Mukhamediev, 1994. Singular points in nonuniform field trajectories of the principle tectonic stress. Physics Solid Earth. 29 (11), 956-965.

[17] Galybin, A.N. \& Sh.A. Mukhamediev, 1999. Plane elastic boundary value problem posed on orientation of principal stresses, JMPS. 47, 2381-2409

[18] Hoppa, G. V., Tufts, B.R., Greenberg, R. and P.E. Geissler, 1999. Formation of Cycloidal Features on Europa. Science. 285, 1899-1902

[19] Sandwell, D.T., Johnson, C.L.. Bilotti, F and J. Suppe, 1997. Driving forces for limited tectonics on Venus. Icarus. 129, 232-244. 